\title{
Versatile Thymine DNA Glycosylase (TDG): Attractive Subject of Future Research and Potential Target for Therapeutics
}

\section{Atanu Maiti*}

Dr. B. C. Guha Centre for Genetic Engineering \& Department of Biotechnology, University of Calcutta, West Bengal, India

*Corresponding author: Maiti A, Dr. B. C. Guha Centre for Genetic Engineering \& Department of Biotechnology, University of Calcutta, 35 Ballygunge Circular Road, Kolkata -700019, West Bengal, India, Tel: 0332241 0071; E-mail: amait28@gmail.com

Received date: July 24, 2014, Accepted date: July 25, 2014, Published date: July 26, 2014

Copyright: (C) 2014 Maiti A, et al. This is an open-access editorial distributed under the terms of the Creative Commons Attribution License, which permits unrestricted use, distribution, and reproduction in any medium, provided the original author and source are credited.

\section{Editorial}

Human Thymine DNA glycosylase or hTDG was first discovered in 1993 in Josef Jiricny's laboratory from HeLa cells extract [1]. Thymine DNA glycosylase was named because of its ability to excise T from G.T mispair which predominantly arise due to spontaneous deamination of 5-methyl cytosine at CpG sites in mammals by AID/APOBEC enzyme [2]. Initially TDG attracted attention due to its DNA repair activity initiating Base Excision Repair (BER) pathway. Participating in BER TDG protect our genome from hazardous mutation. Subsequent research on TDG opens up many new insights of TDG's structure, function and mechanism. Few recent discoveries like, TDG is essential for embryonic development, interacts with many transcription factors and regulates gene expression and its important role in active DNA demethylation brought TDG in limelight of research especially in the field of epigenetic. Thus in mammal TDG is an essential enzyme with multifunctional activity.

TDG plays an important role in maintaining genetic and epigenetic integrity of our genome. In mammals DNMTs convert cytosine to 5methylcytosine $(\mathrm{mC})$ replacing a hydrogen by a methyl group at 5 th carbon of the pyrimidine ring. This conversion is specific at $\mathrm{CpG}$ dinucleotide sequences and thus $\mathrm{CpG}$ sequence is epigenetically important and central for cytosine methylation in mammals [3]. This epigenetic modification is implicated to many biological processes like regulation of gene expression, genomic imprinting, cell fate differentiation, defense against viral gene expression etc. [4,5]. Spontaneous oxidative deamination of $\mathrm{mC}$ by AID/APOBEC, produce highly mutagenic G.T mispair in the genome [2]. Unrepaired G.T mispair lead to $\mathrm{C} \rightarrow \mathrm{T}$ or $\mathrm{C} \cdot \mathrm{G}$ to $\mathrm{T} \cdot \mathrm{A}$ transition mutation at $\mathrm{CpG}$ sites. This mutation not only changes the amino acids codon sequence it also alter the epigenetic sequence $\mathrm{CpG}$ to $\mathrm{TpG}$ which is detrimental for our genetic and epigenetic integrity. It is evident that in different cancer types a significant number of mutations in $\mathrm{P} 53$ gene are $\mathrm{C} \rightarrow \mathrm{T}$ point mutation raise at $\mathrm{CpG}$ site [6-8]. TDG along with another enzyme MBD4, protect our genome from this inevitable mutation. Initiating BER, TDG excise $\mathrm{T}$ from G.T mispair producing an abasic side and follow-on BER process restore a G.C pair. TDG also excise Uracil, 5halo Uracil and many other modified bases when mispaired with Guanine preferentially at CpG site. Though Thymine is consider the biological substrate of TDG, rate of excision of Thymine is lower than 5-halo Uracil and Uracil when mispaired with Guanine. Relatively lower G.T repair activity of TDG questioned its role in DNA repair and could potentially be a reason behind hyper mutation found at CpG site. But, unlike other DNA glycosylase (Except MBD4) TDG removes canonical base $\mathrm{T}$ from $\mathrm{G}$.T mispair avoiding $\mathrm{T}$ from huge excess of $\mathrm{A} \cdot \mathrm{T}$ pairs, which is highly challenging task for a DNA glycosylase and requires very stringent mechanisms. Recent mechanistic investigation showed that few conserved residues of TDG suppress its G.T repair activity. Mutation of those residues increase G.T activity with more increased A.T activity which is unsafe for the genomic stability. These findings suggest, TDG has evolved with the need of suppressed G.T repair activity to avoid aberrant activity on normal A.T pair [9]. Thus TDG balances the need for efficient lesion processing with minimal action on undamaged DNA [10].

DNA methylation is an important epigenetic modification involved in many crucial biological processes. Again, abnormal methylation is implicated in cancer and many developmental disorders [11]. Thus a proper maintenance of DNA methylation is crucial and needs a mechanism of DNA demethylation. The mechanism of DNA demethylation have been proposed as passive, active or a combination of both. Passive DNA demethylation can occur by inhibition or lack of DNA methyltransferases during replication cycles, whereas active DNA demethylation occur through the excision of $\mathrm{mC}$ or modified $\mathrm{mC}$ by specific DNA glycosylases and then restoration of cytosine through BER pathway. Recent findings reveal that TDG plays an important role in active DNA demethylation in mammals. This has been supported by the fact that either knockout or catalytic inactivity of TDG leads to embryonic lethality in mice $[12,13]$. This observation supports the essentiality of TDG for embryonic development and involvement of TDG glycosylase activity in active DNA demethylation. Three possible pathways by which TDG can mediate active DNA demethylation are deamination pathway, hydroxylation-deamination pathway and deamination independent pathway [14]. In all these pathways TDG initiates the BER by excising the modified $\mathrm{mC}$ and follow- on BER restore a G.C pair. In deamination pathway $\mathrm{mC}$ is first deaminated to $\mathrm{T}$ by AID/APOBEC and then $\mathrm{T}$ is excised by TDG. Whereas in hydroxylation-deamination pathway first $\mathrm{mC}$ is oxidized to hmC (hydroxymethyl Cytosine) by Tet (Tet1-3) enzymes and hmC deamnated to hmU (hydroxymethyl Uracil) by AID/APOBEC and then hmU is excised by TDG. Though two other glycosylases, MBD4 and SMUG1 are able to excise $\mathrm{T}$ and hmU respectively, TDG is proficient on both deaminated products [13]. Deamination independent pathway involves the irradiative oxidation of $\mathrm{mC}$ to $\mathrm{hmC}$ to $\mathrm{fC}$ (fromyl Cytosine) to caC (carboxyl Cytosine) by Tet (Tet1-3) enzymes and efficient excision of $\mathrm{fC}$ and $\mathrm{caC}$ by TDG $[15,16]$. So far known, TDG is the only enzyme which can excise $\mathrm{fC}$ and caC. Though more research is needed to establish the actual pathway, recently found substantial reduced activity of AID/APOBEC on $\mathrm{mC}$ and hmC suggest that AID/APOBEC mediated deamination and hydroxylationdeamination pathways are less likely and Tet-mediated oxidation of $\mathrm{mC}$ might be the more potential mechanism for active DNA demethylation [17]. Thus playing a role in active DNA demethylation TDG plays a vital role in maintaining the proper DNA methylation status of our genome. 
Apart from DNA repair and active DNA demethylation, accumulative research supports the fact that TDG plays a central role in regulation of gene expression. This is confirmed by the observations that TDG null mouse or mouse bearing a catalytically inactive TDG variant shows embryonic lethality $[12,13]$. It is evident that TDG interacts with several transcription factors like c-Jun, Retinoic Acid Receptor (RAR), Retinoic X Receptor (RXR), Estrogen Receptor alpha (ER $\alpha)$ and Thyroid Transcription Factor-1 (TTF-1) etc. and various transcriptional co-activators (CBP/P300, SRC1, NCoR3 etc.) to regulate their functions - playing a role as a transcriptional regulator $[14,18,19]$. It is well known that cytosine methylation at CpG dinucleotide is an important factor for gene regulation. CpG dinucleotides are found very frequent at the promoter region of genes and hypermethylation by DNMTs at promoter region resist transcription factor to sit on promoter to activate the gene. In general, hypermethylated promoter silence a gene whereas genes with unmethylated or hypomethylated promoters are active. TDG also interact with DNA methyltransferase DNMT3a and inhibit the methylation activity of DNMT3a. Thus inhibiting methylation activity of DNMT3a, TDG indirectly regulate CpG methylation and hence gene expression $[14,18]$. Direct involvement of TDG in gene regulation is through the active DNA demethylation of DNA.

As TDG is an essential multifunctional enzyme and regulates several biological processes interacting with various important proteins, TDG is an attractive subject for future research and potential target for therapeutics. It is evident that, genetic mutation and/or hyper methylation of $\mathrm{CpG}$ rich promoter sites (mostly tumor suppressor gene) are very frequent in most cancer and tumor cells. Important role of TDG in DNA repair and active DNA demethylation propose its potential involvement in cancer and tumor genesis. Initial evidence indicates that TDG deficiency or loss of TDG activity increases the chance of tumor and cancer development $[14,18]$. Extensive research on identifying TDG polymorphism/variant associated with different cancer would advance our understanding of cancer and could provide potential biomarker for specific cancer. As abnormal methylation is linked to cancer and many developmental disorders, systematic control of DNA methylation would have wide applicability to the study of basic biomedical problems as well as a therapeutic strategy for the treatment of diseases that involve abnormal (hypo/hyper) methylation of DNA. The regulation of methylation could be achieved either by 1) site-specific methylation or by 2) sitespecific demethylation of target genes. Engineered zinc fingermethyltransferase domains have been shown promises for site- specific methylation to a hypomethylated region of genome [20,21]. Similarly engineered TDG could offer site-specific demethylation of target genes which are hypermethylated. Thus development of promoter specific engineered TDG could provide valuable molecular tools for reactivation of different tumor suppressor genes which are silenced in different cancer or tumor cells. Alexey V Fedulov and co-workers were able to demethylate target specifically by fusing TDG with a well characterized DNA binding domain (RHD) of NFkB [22]. This initial evidence provided by Alexey V Fedulov and co-workers suggest the feasibility of this approach. TDG interaction with transcription factors regulates several genes associated with cellular defense, proper development and epigenetic alteration etc. Despite the potential role in human biology, very little studies have been done on TDG transcription factors interaction and their consequences. More research in this area will help to understand the involvement of TDG in different developmental and aging related diseases. It could also help to develop potential therapeutics for the treatment of the same. More and more possibilities will be open up with advancing research on TDG and its interaction with other proteins.

\section{References}

1. Neddermann P, Jiricny J (1993) The purification of a mismatch-specific thymine-DNA glycosylase from HeLa cells. J Biol Chem 268: 21218-21224.

2. Morgan HD, Dean W, Coker HA, Reik W, Petersen-Mahrt SK (2004) Activation-induced cytidine deaminase deaminates 5-methylcytosine in DNA and is expressed in pluripotent tissues: implications for epigenetic reprogramming. J Biol Chem 279: 52353-52360.

3. Klose RJ, Bird AP (2006) Genomic DNA methylation: the mark and its mediators. Trends Biochem Sci 31: 89-97.

4. Feng S, Jacobsen SE, Reik W (2010) Epigenetic reprogramming in plant and animal development. Science 330: 622-627.

5. Deaton AM, Bird A (2011) CpG islands and the regulation of transcription. Genes Dev 25: 1010-1022.

6. Rideout WM 3rd, Coetzee GA, Olumi AF, Jones PA (1990) 5Methylcytosine as an endogenous mutagen in the human LDL receptor and p53 genes. Science 249: 1288-1290.

7. Cooper DN, Youssoufian H (1988) The CpG dinucleotide and human genetic disease. Hum Genet 78: 151-155.

8. Pfeifer GP, Besaratinia A (2009) Mutational spectra of human cancer. Hum Genet 125: 493-506.

9. Maiti A, Noon MS, MacKerell AD Jr, Pozharski E, Drohat AC (2012) Lesion processing by a repair enzyme is severely curtailed by residues needed to prevent aberrant activity on undamaged DNA. Proc Natl Acad Sci U S A 109: 8091-8096.

10. Drohat AC, Pozharski E, Maiti A (2012) How a mismatch repair enzyme balances the needs for efficient lesion processing and minimal action on undamaged DNA. Cell Cycle 11: 3345-3346.

11. Wu SC, Zhang Y (2010) Active DNA demethylation: many roads lead to Rome. Nat Rev Mol Cell Biol 11: 607-620.

12. Cortázar D, Kunz C, Selfridge J, Lettieri T, Saito Y, et al. (2011) Embryonic lethal phenotype reveals a function of TDG in maintaining epigenetic stability. Nature 470: 419-423.

13. Cortellino S, Xu J, Sannai M, Moore R, Caretti E, et al. (2011) Thymine DNA glycosylase is essential for active DNA demethylation by linked deamination-base excision repair. Cell 146: 67-79.

14. Dalton SR, Bellacosa A (2012) DNA demethylation by TDG. Epigenomics 4: 459-467.

15. He YF, Li BZ, Li Z, Liu P, Wang Y, et al. (2011) Tet-mediated formation of 5 -carboxylcytosine and its excision by TDG in mammalian DNA. Science 333: 1303-1307.

16. Maiti A, Drohat AC (2011) Thymine DNA glycosylase can rapidly excise 5formylcytosine and 5-carboxylcytosine: potential implications for active demethylation of CpG sites. J Biol Chem 286: 35334-35338.

17. Maiti A (2012) Mechanism of active DNA demethylation: Recent progress in epigenetics. J Biomol Res Ther 1:e106

18. Sjolund AB, Senejani AG, Sweasy JB (2013) MBD4 and TDG: multifaceted DNA glycosylases with ever expanding biological roles. Mutat Res 743-744: $12-25$.

19. Cortázar D, Kunz C, Saito Y, Steinacher R, Schär P (2007) The enigmatic thymine DNA glycosylase. DNA Repair (Amst) 6: 489-504.

20. Nunna S, Reinhardt R2, Ragozin S1, Jeltsch A1 (2014) Targeted methylation of the epithelial cell adhesion molecule (EpCAM) promoter to silence its expression in ovarian cancer cells. PLoS One 9: e87703.

21. Chaikind B, Ostermeier M2 (2014) Directed evolution of improved zinc finger methyltransferases. PLoS One 9: e96931.

22. Gregory DJ, Mikhaylova L, Fedulov AV (2012) Selective DNA demethylation by fusion of TDG with a sequence-specific DNA-binding domain. Epigenetics 7: 344-349. 